\title{
Monte Carlo Study of Magnetism of the Fe/Gd Multilayers: Dependence on the Layers Thickness and Interface Morphology
}

\author{
Abdelati Razouk, Mohamed Sahlaoui, and Mohamed Sajieddine \\ Laboratoire de Physique et Mécanique des Matériaux, Faculté des Sciences et Techniques, \\ Université Sultan Moulay Slimane, BP 523, 23000 Béni-Mellal, Morocco \\ Correspondence should be addressed to Abdelati Razouk, razoukab@gmail.com \\ Received 3 April 2012; Accepted 15 May 2012 \\ Academic Editors: G. Alfieri and T. I. Shabatina \\ Copyright ( $(2012$ Abdelati Razouk et al. This is an open access article distributed under the Creative Commons Attribution \\ License, which permits unrestricted use, distribution, and reproduction in any medium, provided the original work is properly \\ cited. \\ We present the results of the Monte Carlo simulations of Fe/Gd multilayers and a comparison of the models studied with \\ experimental results. The Heisenberg model interactions are considered for both ferromagnetic and antiferromagnetic cases. \\ At thermal equilibrium magnetization, the Curie temperature is investigated for different thicknesses of Fe layers and interface \\ morphology in $\mathrm{Fe} / \mathrm{Gd}$ multilayers. It turns out that the magnetic properties of the $\mathrm{Fe} / \mathrm{Gd}$ multilayers strongly depend on \\ magnetization amplitude with interface composition, the spatial arrangement of magnetic atoms, and the thickness of Fe layers.
}

\section{Introduction}

In the last 20 years, a lot of effort has been devoted to the determination of the magnetic properties of rare earthtransition metal (RE-TM). In particular RE-Fe amorphous multilayers have attracted considerable attention from the viewpoint of technology and also fundamental research [13].

Magnetic layered systems exhibit various fascinating physical properties including giant magnetoresistance (GMR) [4-10]. These properties not only arouse fundamental interest but also are important because of their possible applications in magnetic storage devices and sensors. For instance, double-layer films consisting of two different magnetic layers, that are, two ferromagnetic materials, or a ferromagnetic, and an antiferromagnetic layer are being used in and magneto-optical media, utilizing the exchange coupling acting between two layers. Phenomena, such as, giant magnetoresistance and interlayer magnetic coupling have stimulated a great theoretical interest and opened new pathways for the design of novel devices by correct choice of materials. The periodic stacking of two distinct ferromagnetic materials gives rise to a variety of magnetic exchange interactions. Magnetic multilayers composed of a rare-earth (RE) element, such as, Gd and a transition metal (TM) like $\mathrm{Fe}, \mathrm{Co}$ and $\mathrm{Ni}$ are interesting example systems. Due to their very different ordering temperatures, magnetic configurations depending on the structural parameters, temperature, and magnetic field may occur. In the case of $\mathrm{Gd} / \mathrm{Fe}$ multilayers the impact of the variation of the growth parameters and thickness of the individual constituting $\mathrm{Fe}$ and Gd layers upon the transport and magnetic properties has been investigated extensively. However, most of these investigations were only carried out in the thickness regime of few monolayers (MLs) [11]. The Fe layer thickness is varied from 70 to $150 \mathrm{~nm}$ and its effect on the structural and magnetic properties of $\mathrm{Fe} / \mathrm{Gd} / \mathrm{Fe}$ sandwich multilayers has been explored. Gd films were found to change from amorphous to polycrystalline at a critical thickness of $20 \mathrm{~nm}$ [12].

This paper is organised as follows. In Section 2, we introduce the Heisenberg model and Monte Carlo (MC) method is also described. In Section 3, we show numerical results in comparison with the previous results and the experimental data. Section 4 is devoted to summary and concluding remarks. 


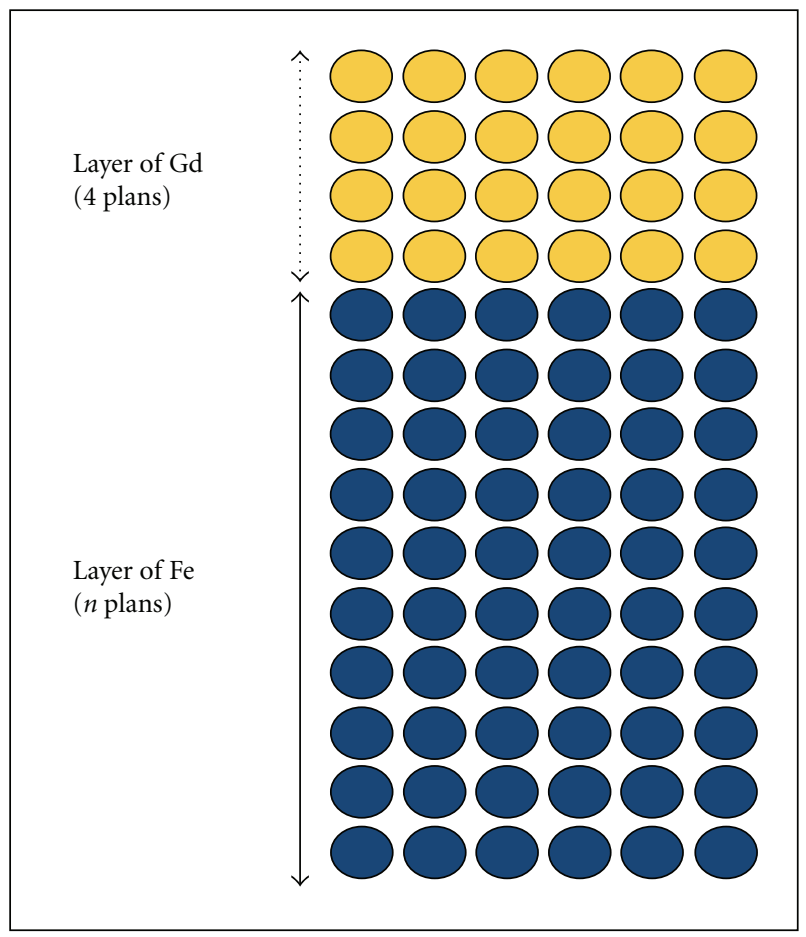

Figure 1: Schematic representation of two-dimensional cross-section through the Fe/Gd bilayers with abrupt interface.

\section{Simulation Method}

Our starting point is a classical Heisenberg model. The Hamiltonian is given in the form:

$$
H=-\sum_{\langle i, j\rangle}^{N} J_{i j}\left(S_{i}^{x} S_{j}^{x}+S_{i}^{y} S_{j}^{y}+S_{i}^{z} S_{j}^{z}\right)
$$

where $N$ is the number of sites (spins), $S_{i}^{x}, S_{i}^{y}$ and $S_{i}^{z}$ are spin operators following the axes of space OX, OY, and OZ respectively, at site $i$ and sum is taken over nearest-neighbour pair of spins. $J_{i j}$ denotes nearest-neighbour exchange interaction between $S_{i}$ and $S_{j}$ spins.

Where $\vec{S}_{i}$ is the classical Heisenberg spin, $J_{i j}$ is the nearestneighbour exchange interaction sites $i$ and $j$. We define the magnetisation as follows:

$$
M(T)=\frac{\left\langle\left[\left(\sum_{i} m_{i}^{x}\right)^{2}+\left(\sum_{i} m_{i}^{y}\right)^{2}+\left(\sum_{i} m_{i}^{z}\right)^{2}\right]^{1 / 2}\right\rangle_{T}}{N}
$$

where the $N(N=L \times L \times n)$ is the number of atoms, $m_{i}^{x}=$ $-g_{i} S_{i}^{x} /\left(g_{i}-1\right)$ is the $x$ component of the moment of the atom $i$ in Bohr magneton units, and \langle\rangle$_{T}$ means statistical average at temperature $T$.

The flowing magnetic parameters are those of free atoms magnetic: $g_{\mathrm{Gd}}=g_{\mathrm{Fe}}=2, S_{\mathrm{Fe}}=1$, and $S_{\mathrm{Gd}}=7 / 2$. The exchange interactions have been adjusted to obtain pure polycrystalline Fe and Gd Curie temperature (see [4]).
For the numerical analysis of the magnetic properties of Fe/Gd multilayers described above, we have used the Monte Carlo simulations with the Metropolis algorithm [13, 14]. The Metropolis Monte Carlo algorithm enables one to obtain the macrostate equilibrium for a physical system at the given temperature $T$. The basic idea of this method consists of the following procedure: the spins are examined individually. A site $i$ is randomly chosen and a unit vector defined by the random choice with uniform distribution of its $z$-component $z_{i} \in[-1,1]$ and its azimuthal angle $\phi_{i} \in[0,2 \pi[$ is determined.

It has to be noted that this spin trial rotation procedure is isotropic. Then the energy variation $\Delta E$ associated to this rotation is calculated. The next step is the following:

(i) if $\Delta E \leq 0$, the rotation is accepted;

(ii) if $\Delta E>0$, the rotation may be accepted with a probability that is proportional to the Boltzmann factor $\exp \left(\Delta E / k_{\mathrm{BT}}\right)$ in order to take into account the thermal fluctuations.

One MC step consists in examining all spins of the system once. At each temperature, IT0 MC steps were performed to reach the thermodynamic equilibrium, and afterwards the physical quantities were measured by averaging over the next ITF MC steps.

It is important to note that finite size effects have no significant influence on the physical properties under consideration. Only nearest-neighbour interactions should be taken into account since magnetic studies of such systems in 

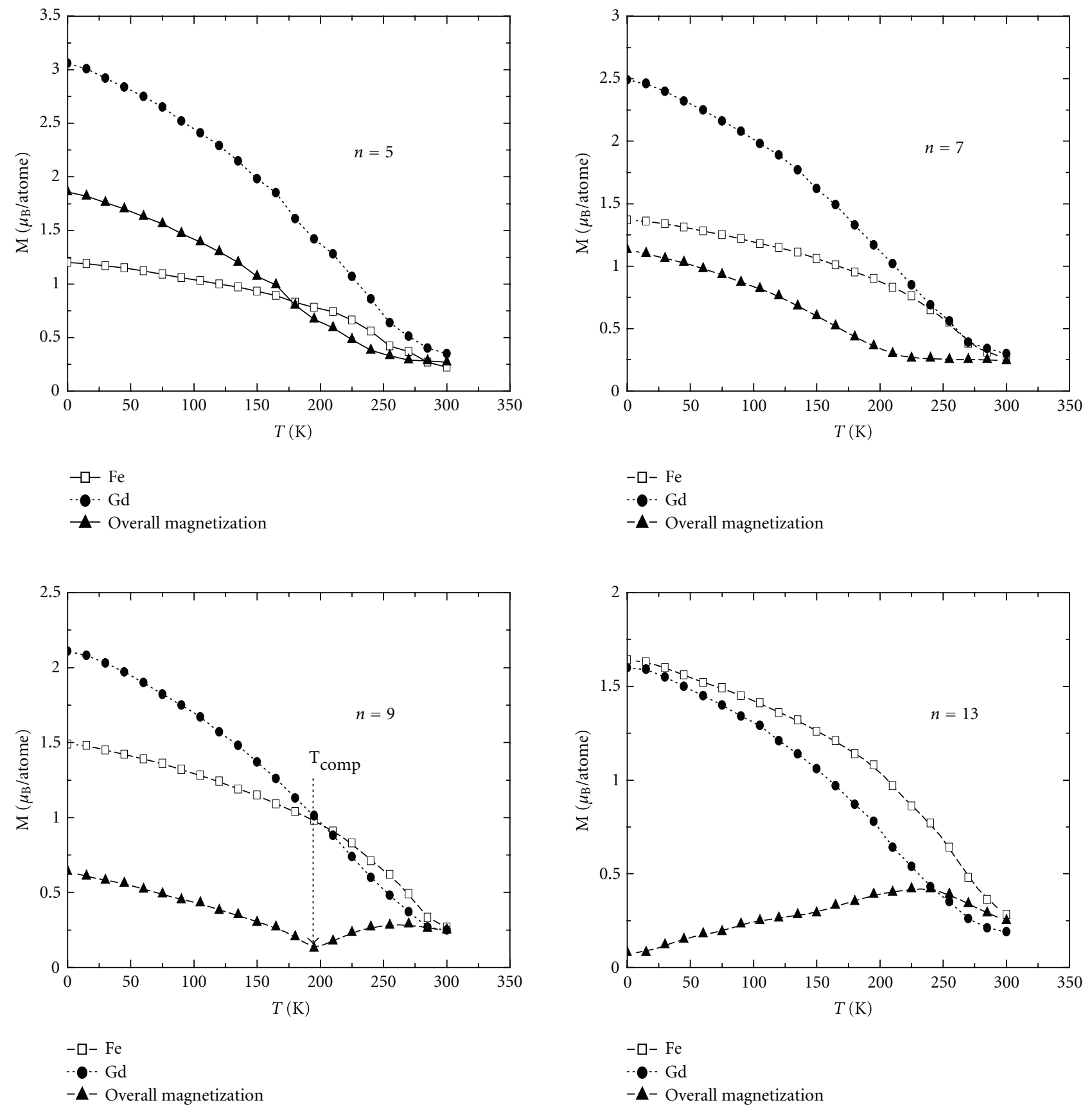

Figure 2: Thermal variations of magnetization and subnets $\mathrm{Fe}$ and $\mathrm{Gd}$ for $\mathrm{Fe}_{n} / \mathrm{I} / \mathrm{Gd}_{4}$ multilayers with abrupt interface.

particular in amorphous alloys show evidence that the value of the next nearest-neighbour interactions is one order of magnitude smaller [15]. Indeed, the exchange interactions are very strong in transition metal but at short range.

\section{Results}

To obtain a simulation model suitable for multilayer Fe/Gd with most realistic cases (diffuse interface), we consider the case of $\mathrm{Fe}_{n} / \mathrm{Gd}_{m}$ multilayers without anisotropy; the number of planes $(m)$ of the Gd layer is kept constant to 4 while the number of planes $(n)$ of the Fe layer varies from 1 to 13 .

Our approach is to study the multilayers with (i) abrupt interface $\left(\mathrm{Fe}_{n} / \mathrm{Gd}_{m}\right)$. These results provide a basis for interpreting the magnetic properties of real case with diffuse interface $\mathrm{Fe}_{n} / \mathrm{I} / \mathrm{Gd}_{m}$; (ii) modulated interface $\mathrm{Fe}_{n} / \mathrm{I} / \mathrm{Gd}_{m}$ on 2 planes ( 1 plane of $\mathrm{Fe}+1$ plan of $\mathrm{Gd}$ ).

3.1. Case of Abrupt Interface. Figure 1 shows a representation of a multilayer abrupt interface that will be used to interpret 


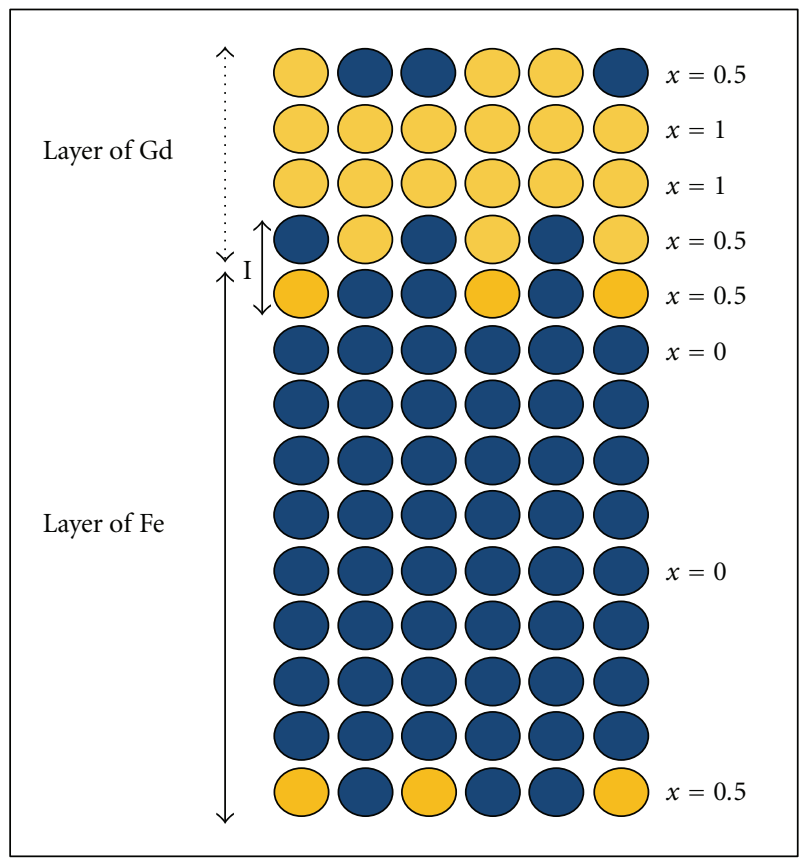

FIGURE 3: Schematic representation of two-dimensional cross-section through the Fe/I/Gd bilayers with interface-modulated two atomic planes.

results obtained for modulated interfaces. We are interested in the following to study the influence of the iron thickness on the magnetic properties of multilayers Fe/Gd. The number of planes of the individual layers corresponding to thickness of iron $e_{\mathrm{Fe}}$ is between 13 and $33 \AA$ and the gadolinium thickness is $e_{\mathrm{Gd}}=16 \AA$. The different physical parameters and simulation are summarized in Table 1 . The interface exchange interaction $J_{\mathrm{Fe}-\mathrm{Gd}}(x)$ was determined from the following equation [16]:

$$
J_{\mathrm{Fe}-\mathrm{Gd}}=-\frac{J_{\mathrm{Fe}-\mathrm{Fe}}+J_{\mathrm{Gd}-\mathrm{Gd}}}{2} .
$$

Figure 2 shows the thermal variations of magnetization of the whole sample and the sublayers of Fe and Gd for different $\mathrm{Fe}$ thickness. We find that for small thicknesses of Fe $\left(n \leq n_{c}=7, n_{c}\right.$ depends mainly on the number of Gd planes), the magnetization of Gd layer dominates over the selected temperature range. For larger thickness $(7<n<13)$, the magnetization of Fe layer dominates at high temperatures and the Gd layer dominates at low temperatures and compensation between the magnetizations of two sublattices is observed. For larger thicknesses of $\mathrm{Fe}$ ( $n \geq 13$ ), the Fe layer dominates whatever the temperature. In addition, the compensation temperature decreases. These results are qualitatively consistent with experimental results for multilayers Fe/Gd [17] which provide $T_{\text {comp }} \leq 300 \mathrm{~K}$.

The observation of magnetization curves by atomic plane and type of atoms shows that spins of the heart of Fe layer are arranged more quickly than those of interface where $\left\langle Z_{\mathrm{Fe}-\mathrm{Fe}}\right\rangle$ is lower.
TABLe 1: Physical and simulation parameters used to study Fe/Gd bilayer with abrupt interface.

\begin{tabular}{lccc}
\hline \multicolumn{2}{c}{ Physical parameters } & \multicolumn{2}{c}{ Simulation parameters } \\
\hline$J_{\mathrm{Fe}-\mathrm{Fe}}-J_{\mathrm{Fe}-\mathrm{Fe}}$ & $110 \mathrm{~K}$ & $N$ (spins) & $900-1600$ \\
$J_{\mathrm{Fe}-\mathrm{Gd}}$ & $-58 \mathrm{~K}$ & IT0 & $2.5 \times 10^{3}-4 \times 10^{3}$ \\
$J_{\mathrm{Gd}-\mathrm{Gd}}$ & $7.5 \mathrm{~K}$ & ITF & $2.5 \times 10^{4}-5 \times 10^{4}$ \\
\hline
\end{tabular}

TABle 2: Physical and simulation parameters used to study $\mathrm{Fe}_{n} / \mathrm{I} / \mathrm{Gd}_{4}$ bilayer with interface modulated on two atomic planes of type $\mathrm{Fe}_{0.5} \mathrm{Gd}_{0.5}$.

\begin{tabular}{lccc}
\hline \multicolumn{2}{c}{ Physical parameters } & \multicolumn{2}{c}{ Simulation parameters } \\
\hline$J_{\mathrm{Fe}-\mathrm{Fe}}$ & $110 \mathrm{~K}$ & $N$ (spins) & $900-1600$ \\
$J_{\mathrm{Gd}-\mathrm{Gd}}$ & $7.5 \mathrm{~K}$ & IT0 & $2.5 \times 10^{3}-4 \times 10^{3}$ \\
$J_{\mathrm{Fe}-\mathrm{FeI}}=J_{\mathrm{Fe}-\mathrm{Fe}}(0.5)$ & $530 \mathrm{~K}$ & ITF & $2.5 \times 10^{4}-5 \times 10^{4}$ \\
$J_{\mathrm{Fe}-\mathrm{GdI}}=J_{\mathrm{Fe}-\mathrm{Gd}}(0.5)$ & $-49 \mathrm{~K}$ & - & - \\
\hline
\end{tabular}

3.2. Modulated Interface. We begin firstly this study with a bilayer whose interface is a homogeneous alloy with a composition as $\mathrm{Fe}_{0.5} \mathrm{Gd}_{0.5}\left(x_{\mathrm{Gd}}=0.5\right)$ which is about on two atomic planes. Thus a bilayer contains three different magnetic zones: 2 pure planes of $\mathrm{Gd}$, at least one pure plane of Fe and a mixed area (Figure 3). This is an improvement over the case of abrupt interfaces.

The physical parameters and simulation parameters are listed in Table 2. The exchange interactions in the interface are defined in Figure 4.

Into a pure plane and between two planes of $\mathrm{Fe}$, the interaction $J_{\mathrm{Fe}-\mathrm{Fe}}$ is constant. Between a pure plane of Fe and 


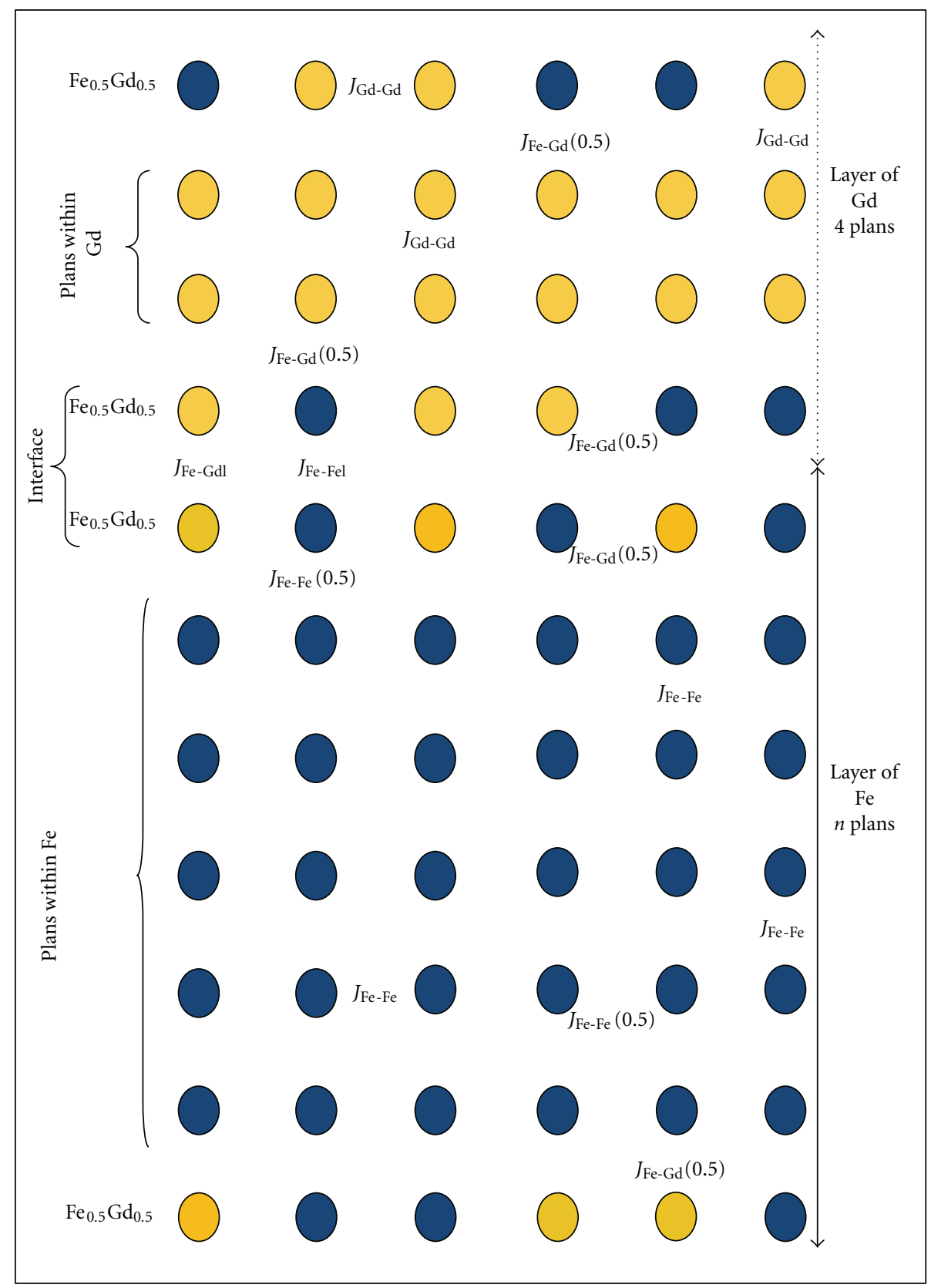

FIGURE 4: Schematic representation of the exchange interactions of the $\mathrm{Fe}_{n} / \mathrm{I} / \mathrm{Gd}_{4}$ bilayers with interface modulated with two atomic planes $x_{\mathrm{Gd}}=0.5$ at the interface.

a plane of the interface, we use the interactions $J_{\mathrm{Fe}-\mathrm{FeI}}=$ $J_{\mathrm{Fe}-\mathrm{Fe}}(0.5)$ and $J_{\mathrm{Fe}-\mathrm{GdI}}=J_{\mathrm{Fe}-\mathrm{Gd}}(0.5)$, with $J_{\mathrm{Fe}-\mathrm{FeI}}$ and $J_{\mathrm{Fe}-\mathrm{GdI}}$ are the interactions used in the abrupt interface.

Total and sublayers magnetization curves are showed in Figure 5. We note that the magnetization of Gd layer dominates at all temperatures as $n<12$. Magnetic compensation exists for $n$ close to 12 where $T_{\text {comp }}=150 \mathrm{~K}$. In fact the spins at the interface are ordered firstly (in opposition to abrupt interfaces case).
Next, a bilayer with a modulated interface of $x_{\mathrm{Gd}}=0.75$ is considered. This is to introduce a more realistic composition modulation obtained with a plane rich in $\mathrm{Fe}$ atoms on the side of iron layer and a plane rich in $\mathrm{Gd}$ atoms beside of $\mathrm{Gd}$ layer. Thus, in our calculations, the interface (I) is composed with two atomic planes; $\mathrm{Fe}_{0.25} \mathrm{Gd}_{0.75}$ and $\mathrm{Fe}_{0.75} \mathrm{Gd}_{0.25}$. Table 3 covers all concentrations $x$ for each plane of the bilayer $\mathrm{Fe}_{n} / \mathrm{I} / \mathrm{Gd}_{4}$, the values of exchange interactions used in each atomic plane and different transition temperatures for iron, 

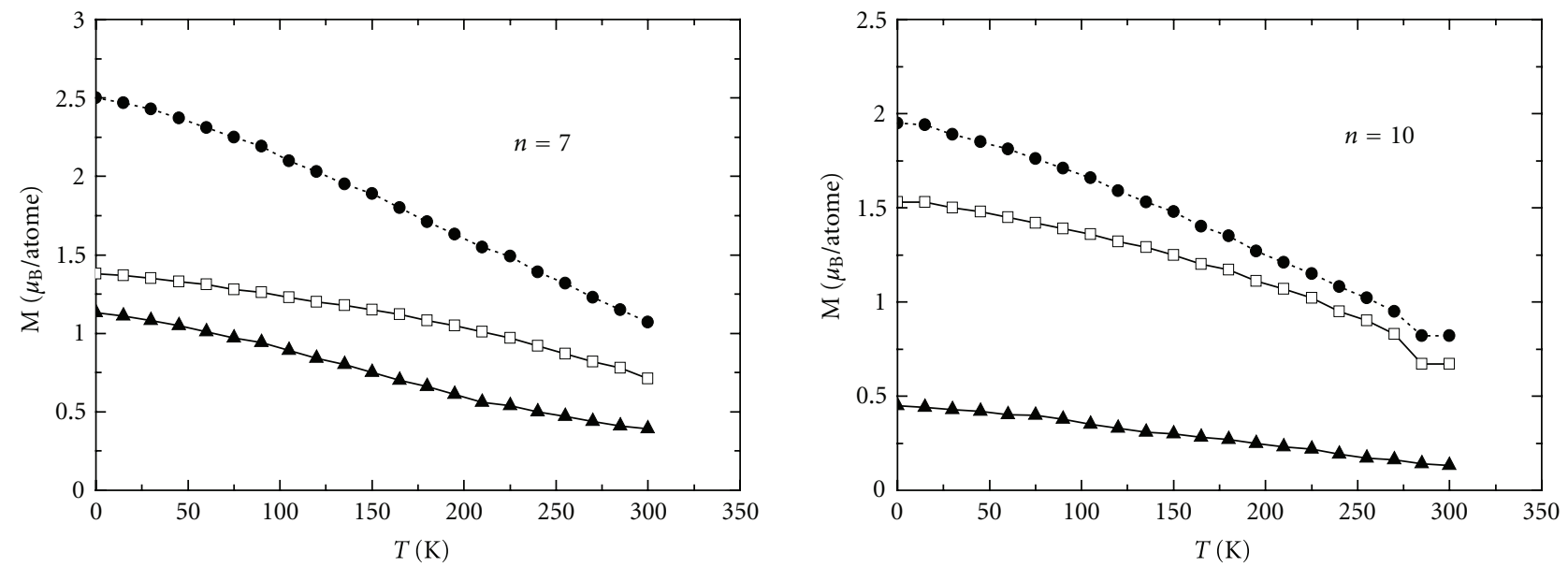

$$
\begin{aligned}
& -\square-\mathrm{Fe} \\
& --\cdot \mathrm{Gd} \\
& - \text { - Overall magnetization }
\end{aligned}
$$

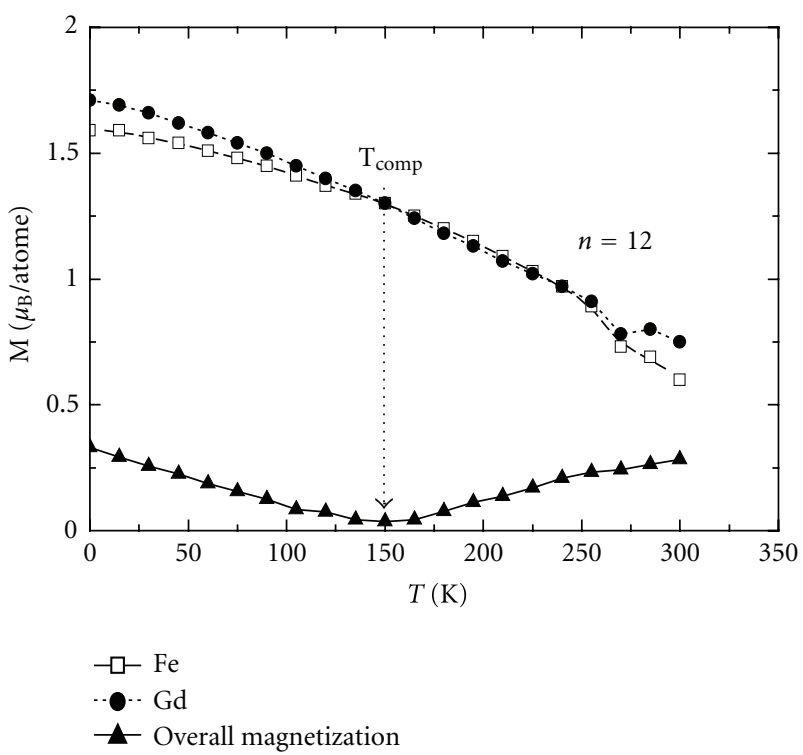

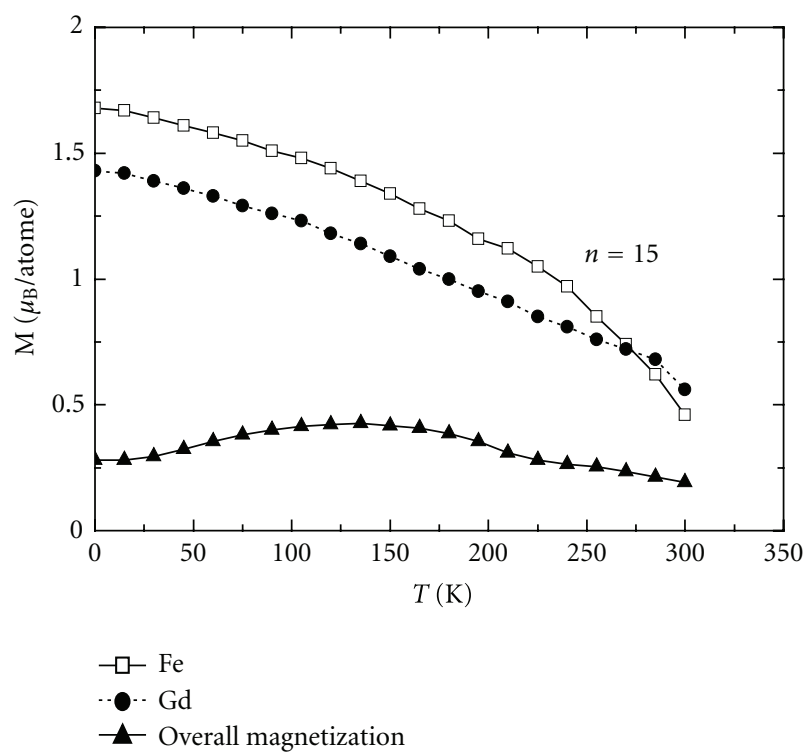

FIGURE 5: Thermal variations of magnetization and subnets $\mathrm{Fe}$ and $\mathrm{Gd}$ for $\mathrm{Fe}_{n} / \mathrm{I} / \mathrm{Gd}_{4}$ multilayers with modulated interfaces and $x_{\mathrm{Gd}}=0.5$.

gadolinium, alloys $\mathrm{Fe}_{0.25} \mathrm{Gd}_{0.75}$ and $\mathrm{Fe}_{0.75} \mathrm{Gd}_{0.25}$. Exchange interactions are defined in Figure 6. In pure Fe planes, $J_{\mathrm{Fe}-\mathrm{Fe}}(0)=J_{\mathrm{Fe}-\mathrm{Fe}}$. Adjustable interactions $J_{\mathrm{Fe}-\mathrm{FeI}}$ and $J_{\mathrm{Fe}-\mathrm{GdI}}$ are chosen according to the atomic concentrations found in different planes: $J_{\mathrm{Fe}-\mathrm{FeI}}=532 \mathrm{~K}$ and $J_{\mathrm{Fe}-\mathrm{GdI}}=-50 \mathrm{~K}$.

Figure 7 shows the evolution of the global magnetization of the bilayer and sublattices. The magnetization curve on $n=11$ shows a minimum at about $180 \mathrm{~K}$; this minimum slightly marked is reflecting a strong influence of the interface on the magnetization of the bilayer. This is clearly seen on the values of the magnetization compared to the case $x_{\mathrm{Gd}}=$ 0.5: In this study, the magnetization at low temperature is
TABLE 3: Values of exchange interactions and $T_{C}$ of the pure elements $\mathrm{Fe}$ and $\mathrm{Gd}$ alloys and $\mathrm{Fe}_{0.75} \mathrm{Gd}_{0.25}$ and $\mathrm{Fe}_{0.25} \mathrm{Gd}_{0.75}$.

\begin{tabular}{lccc}
\hline$x_{\mathrm{Gd}}$ & $J_{\mathrm{Fe}-\mathrm{Fe}}$ & $J_{\mathrm{Fe}-\mathrm{Gd}}$ & $T_{C}(16 \times 10 \times 10)$ \\
\hline 0 & $110 \mathrm{~K}$ & - & $202 \mathrm{~K}$ \\
0.25 & $532 \mathrm{~K}$ & $-50 \mathrm{~K}$ & $490 \mathrm{~K}$ \\
0.75 & $230 \mathrm{~K}$ & $-59 \mathrm{~K}$ & $225 \mathrm{~K}$ \\
1 & - & - & $200 \mathrm{~K}$ \\
\hline
\end{tabular}

weaker compared to the cases $n \geq 9$. Note also that the compensation between the magnetization of Fe layer and 


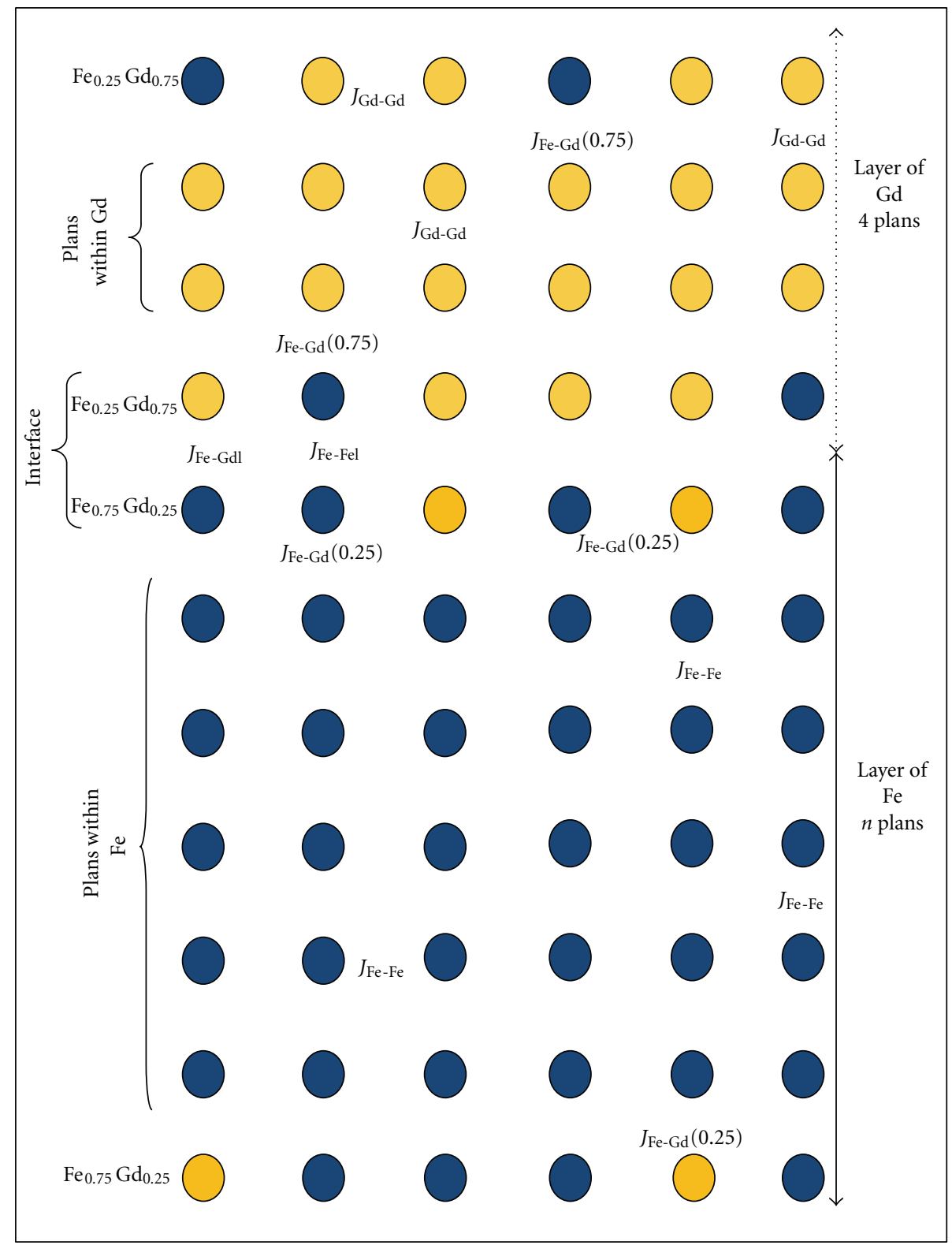

FIGURE 6: Schematic representation of the exchange interactions of the $\mathrm{Fe}_{n} / \mathrm{I} / \mathrm{Gd}_{4}$ bilayres with interface modulated with two atomic planes $\mathrm{Fe}_{1-x} \mathrm{Gd}_{x}$.

that of $\mathrm{Gd}\left(T_{\text {comp }}=180 \mathrm{~K}\right)$ is obtained by adjusting the number of planes of the layer of Fe $(n=11$ instead of 12 for $x_{\mathrm{Gd}}=0.5$ ).

\section{Discussion}

In order to find a simulation model suitable for amorphous multilayer Fe/Gd, we have shown in Figure 8 the variation of transition temperature $\left(T_{C}\right)$ of simulated Fe/Gd multilayers amorphous alloys as a function of equivalent number of terms of Fe and the experimental case.

For multilayer Fe/Gd with abrupt interfaces the transition temperature $T_{C}$ increases with the number of plan Fe and tends to the temperature limit of $200 \mathrm{~K}$ corresponding to amorphous iron from about 11 atomic planes. For low Fe layer thickness, the decrease in $T_{C}$ is due to the decrease in the average coordination number as $n$ decreases. The simulated transition temperatures are well below the transition temperatures of the experimental case. In addition, the change in $T_{C}\left(n_{\mathrm{Fe}}\right)$ is not the same: $T_{C}$ simulated increases when the number of planes of iron increases, which is in contrast with the experimental results for multilayer Fe/Gd [17] and amorphous alloys $\mathrm{Fe}_{1-x} \mathrm{Gd}_{x}$ [18]. This disagreement is attributed to the strong influence of chemical disorder at the interfaces on $T_{C}$. For a multilayer Fe/I/Gd with diffuse interfaces and $x_{\mathrm{Gd}}=0.5$, we find that the presence of the 

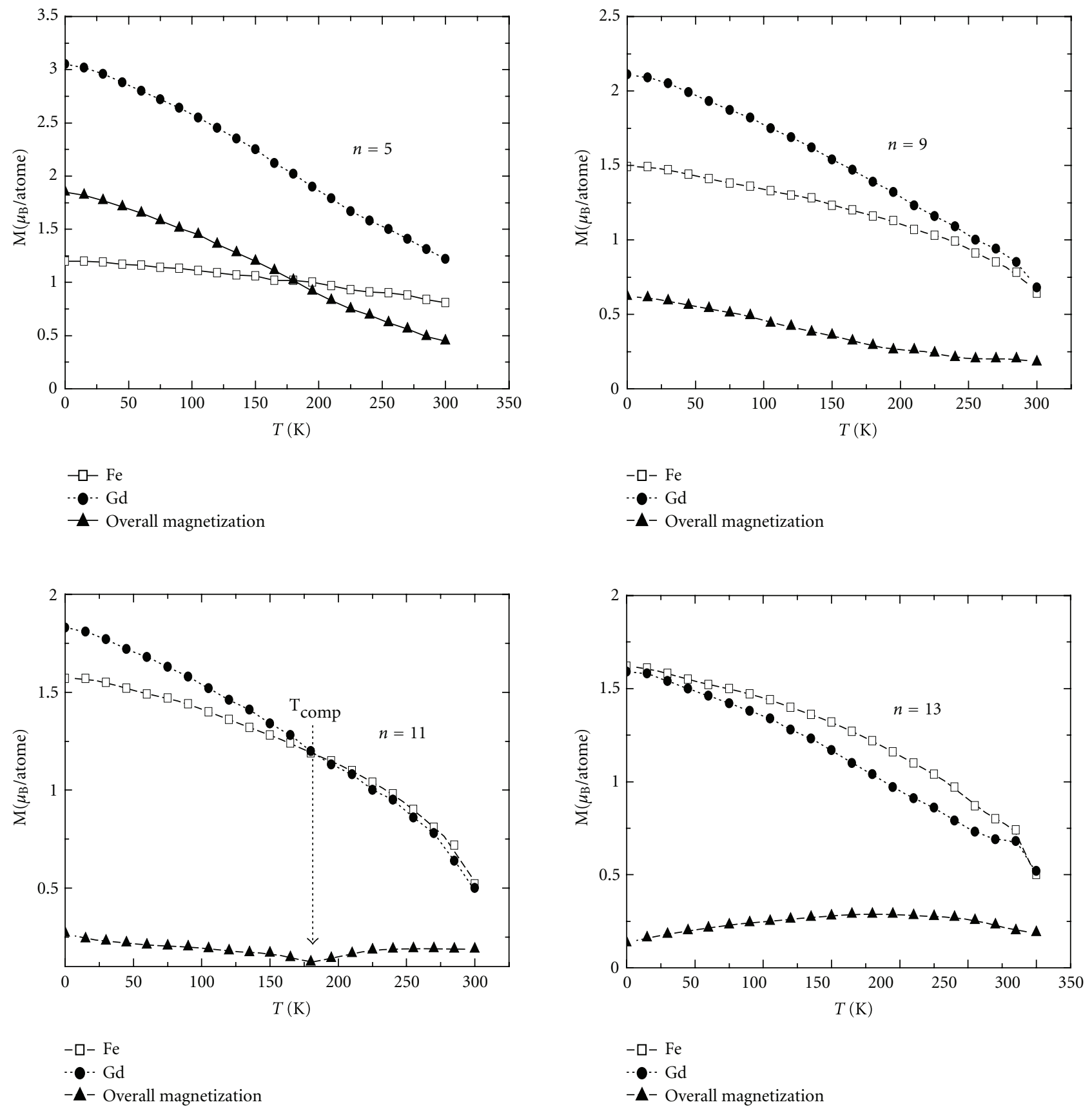

Figure 7: Thermal variations of magnetization and subnets $\mathrm{Fe}$ and $\mathrm{Gd}$ for $\mathrm{Fe}_{n} / \mathrm{I} / \mathrm{Gd}_{4}$ multilayers with modulated interfaces.

modulated interface induces an increase in $T_{C}$ compared to systems with abrupt interface. Unlike the abrupt interface, the transition temperature decreases with increases $n_{\mathrm{Fe}}$ in agreement with experiment [18]. However, the simulated values of $T_{C}$ are still too low compared to the experience.

For a multilayer Fe/I/Gd with $x_{\mathrm{Gd}}=0.75$, the simulated transition temperature is in good agreement with experiment. The simulated values are also close of the results of Nawate et al. [17] but lower than the experimental values of homogeneous alloys Fe-Gd [18]. The difference between simulated results and those of the alloys is still relatively large.

\section{Conclusion}

In summary, we have studied the dependence of magnetic properties on the interface morphology in the Heisenberg multilayers Fe/Gd by using Monte Carlo simulations. Our simulations confirm that the form of distribution of the 


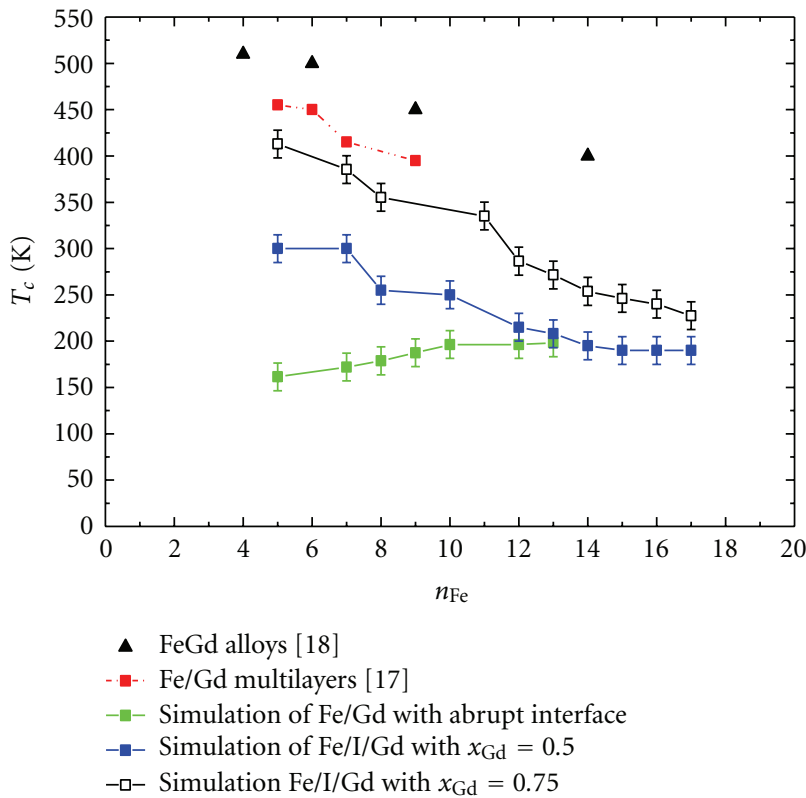

FIGURE 8: Variation of transition temperature $\left(T_{C}\right)$ for experimental and simulated $\mathrm{Fe}_{n} / \mathrm{Gd}_{4}$ bilayers versus the number of $\mathrm{Fe}$ planes.

atoms in interface, that is, morphology interface and thickness of Fe modify its magnetic properties and consequently the magnetic properties of the multilayers. It shows that the results are in good agreement with some qualitative and quantitative experimental results.

\section{References}

[1] J. G. LePage and R. E. Camley, "Surface phase transitions and spin-wave modes in semi-infinite magnetic superlattices with antiferromagnetic interfacial coupling," Physical Review Letters, vol. 65, pp. 1152-1155, 1990.

[2] R. E. Camley, "Surface spin reorientation in thin Gd films on Fe in an applied magnetic field," Physical Review B, vol. 35, pp. 3608-3611, 1987.

[3] R. E. Camley and D. R. Tilley, "Phase transitions in magnetic superlattices," Physical Review B, vol. 37, no. 7, pp. 3413-3421, 1988.

[4] A. Razouk, M. Sahlaoui, M. Sajieddine, and E. Agouriane, "Magnetic properties of Fe1-xGdx amorphous alloys: Monte Carlo investigation," Ferroelectrics, vol. 372, no. 1, pp. 31-35, 2008.

[5] J. Unguris, R. J. Celotta, and D. T. Pierce, "Observation of two different oscillation periods in the exchange coupling of Fe/Cr/Fe(100)," Physical Review Letters, vol. 67, pp. 140-143, 1991.

[6] Y. Chen, A. Malachias, J. Monch, R. Kaltofen, and O. G. Schmidt, "Photoresist-buffer-enhanced antiferromagnetic coupling and the giant magnetoresistance effect of $\mathrm{Co} / \mathrm{Cu}$ multilayers," Journal of Physics, vol. 20, Article ID 452202, 2008.

[7] J. P. Andres, L. Chico, J. Colino, and J. M. Riveiro, "Magnetic behavior of sputtered Gd/Co multilayers," Physical Review B, vol. 66, Article ID 094424, 2002.
[8] M. F. Smith, "Add to my research Anomalous interlayer magnetoresistance in bilayer crystals," Journal of Physics, vol. 24, Article ID 115702, 2012.

[9] J. Q. Xiao, J. S. Jiang, and C. L. Chien, "Giant magnetoresistance in nonmultilayer magnetic systems," Physical Review Letters, vol. 68, pp. 3749-3752, 1992.

[10] F. Petroff, A. Barthélémy, A. Hamzić et al., "Magnetoresistance of Fe/Cr superlattices," Journal of Magnetism and Magnetic Materials, vol. 93, pp. 95-100, 1991.

[11] T. Morishita, Y. Togami, and K. Tsushima, "Magnetism and structure of compositionally modulated Fe-Gd thin films," Journal of the Physical Society of Japan, vol. 54, p. 37, 1985.

[12] K. M. Kant, C. R. H. Bahl, N. Pryds, A. Smith, and J. Schou, "Structural and magnetic properties of $\mathrm{Gd} / \mathrm{Fe}$ multilayers grown by pulsed laser deposition," Journal of Optoelectronics and Advanced Materials, vol. 12, no. 3, pp. 576-579, 2010.

[13] N. Metropolis, A. W. Rosenbluth, M. N. Rosenbluth, A. H. Teller, and E. Teller, "Equation of state calculations by fast computing machines," Journal of Chemical Physics, vol. 21, no. 6, pp. 1087-1092, 1953.

[14] K. Binder, Monte Carlo Methods in Statistical Physics, Springer, New York, NY, USA, 1979.

[15] S. N. Kaul, "Low-temperature magnetization and spin-wave excitations in amorphous Ni-rich transition-metal-metalloid alloys," Physical Review B, vol. 27, pp. 5761-5774, 1983.

[16] L. Veiller, Étude de multicouches Fer/Terbium : Simulation numérique Monte Carlo de propriétés magnétiques et analyse structure par sonde atomique [Ph.D. thesis], Université de Rouen, Rouen, France, Octobre 1998.

[17] M. Nawate, H. Kiriake, and S. Honda, "Spin structures of Gd/Fe multilayers evaporated at oblique incidence," Journal of Magnetism and Magnetic Materials, vol. 121, no. 1-3, pp. 144147, 1993.

[18] P. Hansen, C. Clausen, G. Much, M. Rosenkranz, and K. Witter, "Magnetic and magneto-optical properties of rareearth transition-metal alloys containing Gd, Tb, Fe, Co," Journal of Applied Physics, vol. 66, no. 2, pp. 756-767, 1989. 

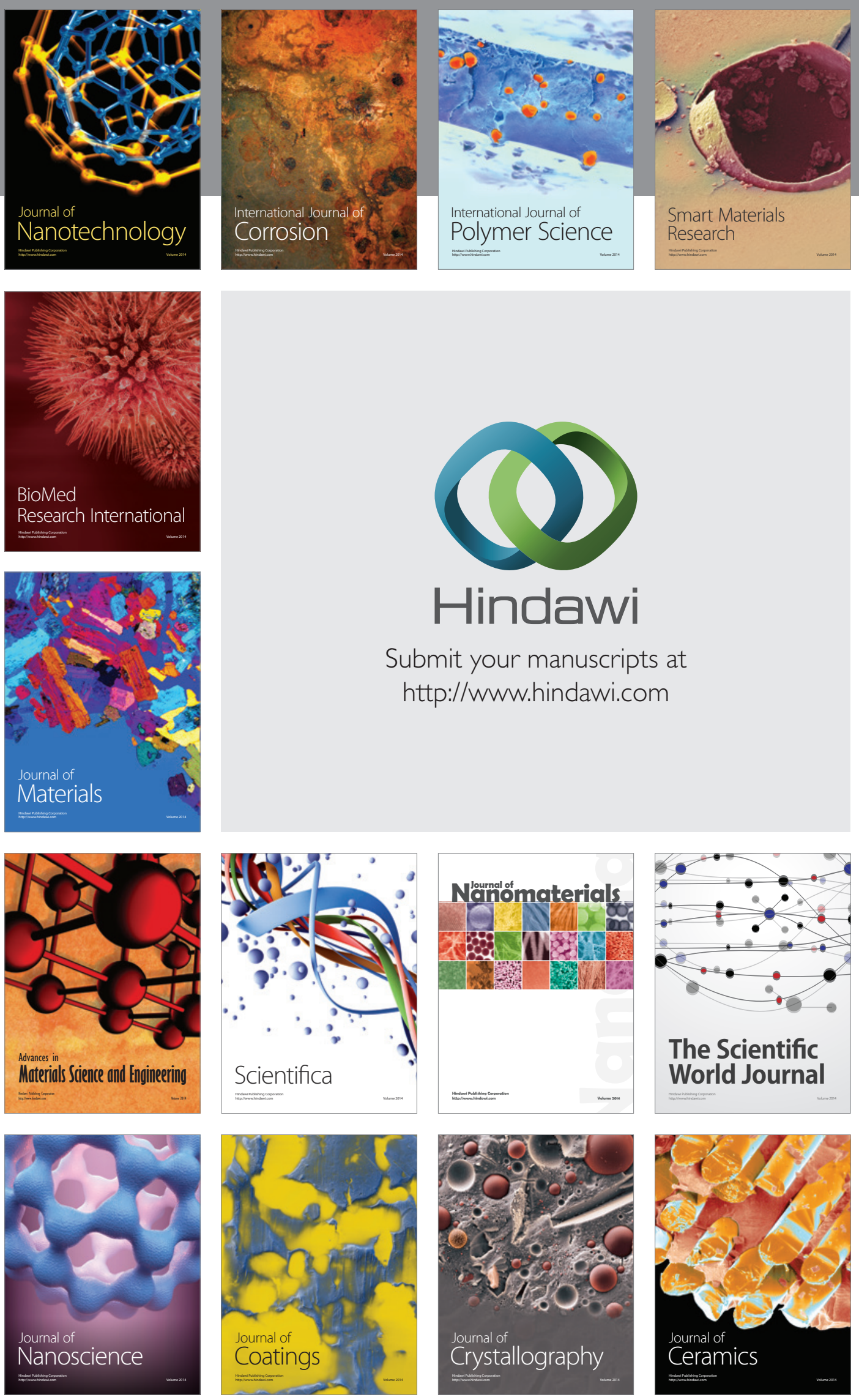

The Scientific World Journal

Submit your manuscripts at

http://www.hindawi.com

\section{World Journal}

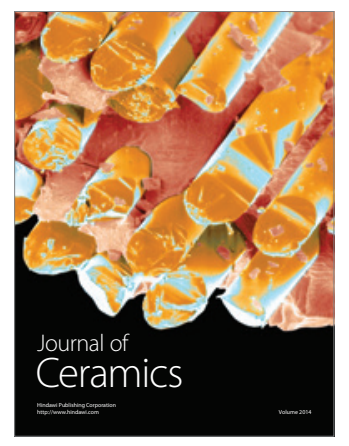

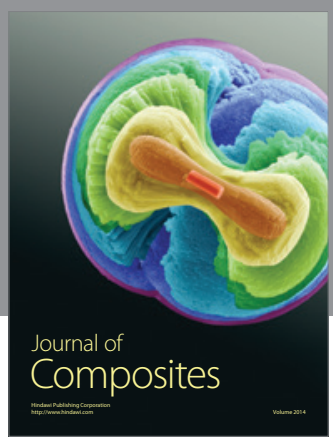
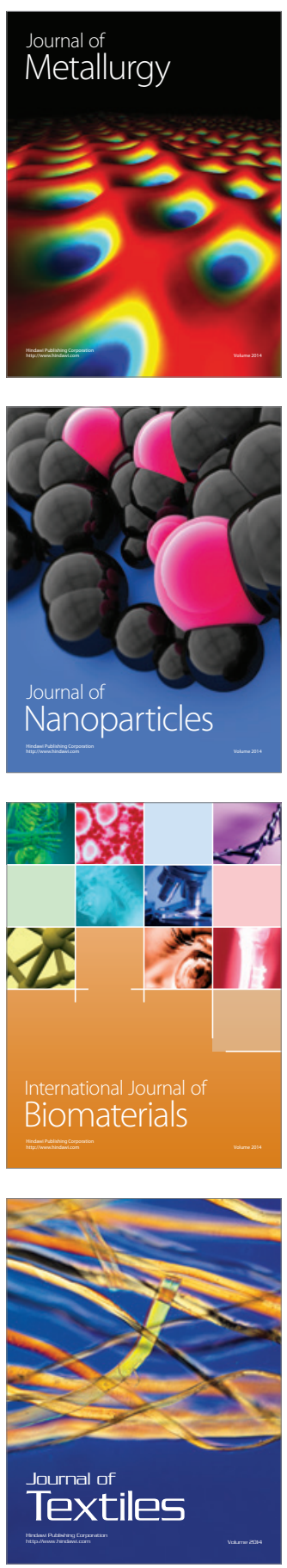